\title{
Potencial ecoturístico en Áreas Naturales Protegidas. Caso: Reserva Biológica Tirimbina, Sarapiquí, Provincia de Heredia, Costa Rica
}

\author{
Ecotourism potential in Protected Natural Areas. Case: \\ Tirimbina Biological Reserve (RBT), Sarapiquí, Province of \\ Heredia, Costa Rica
}

\author{
Mario Arroyo-Solórzano ${ }^{1}$ \\ Universidad de Costa Rica, Costa Rica \\ Lincenth Rojas-Prendas ${ }^{2}$ \\ Universidad Hispanoamericana de Costa Rica, Costa Rica
}

\begin{abstract}
Resumen
En este artículo se describen los principales potenciales turísticos de la Reserva Biológica Tirimbina (RBT). Se determina un nivel de potencial ecoturístico con base en un análisis de variables de importancia y a partir de la descripción de las características de la RBT. La Reserva cuenta actualmente con múltiples programas de investigación natural y educativa, así como con una oferta llamativa de recorridos que propician la interacción y el aprendizaje del turista en la naturaleza. Con base en la metodología de ponderación propuesta se identificaron seis variables relevantes, las cuales se evaluaron con aspectos clave y se les asignó pesos específicos obteniéndose un potencial ecoturístico Alto para la RBT, siendo el senderismo la riqueza natural que se encuentra con mejor ponderación de los aspectos evaluados. La Reserva es fuente de empleo para los habitantes de la zona, contribuye a la protección ambiental, la educación y es un gran impulso para el turismo, con potencial para ligarse a otros encadenamientos turísticos más consolidados.
\end{abstract}

1 Licenciado en Geografía y Geología. Escuela Centroamericana de Geología y Red Sismológica Nacional, Universidad de Costa Rica. Apdo. 214-2060, San Pedro, Costa Rica.

Correo: mario.arroyosolorzano@ucr.ac.cr. (ID https://orcid.org/0000-002-1653-2680

2 Estudiante de Turismo y Hotelería, Universidad Hispanoamericana de Costa Rica, Heredia, Costa Rica. Correo: lincenth28rp@gmail.com. (D) https://orcid.org/0000-0002-3677-1742 
Mario Arroyo-Solórzano Lincenth Rojas-Prendas Ecotourism potential in Protected Natural Areas. Case: Tirimbina Biological Reserve (RBT), Sarapiquí, Province of Heredia, Costa Rica.

Palabras clave: Potencial turístico; Ecoturismo; Reserva Biológica Tirimbina (RBT), Educación ambiental.

\begin{abstract}
This article describes the main tourism potentials of the Reserva Biológica Tirimbina (RBT) (translated as Tirimbina Biological Reserve). The ecotourism potential level is determined based on an analysis of important variables and from the descriptive characteristics of RBT. Currently, RBT sustains multiple nature and educational research programs, as well as a striking offering of tours that promote tourist interaction and learning in nature-based settings. According to the proposed weighting methodology, where six relevant variables were identified and each variable evaluated based on key attributes assigned individual corresponding weight values, RBT is characterized as possessing a high ecotourism potential wherein ecological trail hiking activities predominates over all evaluated attributes. The reserve is a source of employment for the local population, promotes environmental protection and education, and greatly boosts tourism with the potential to link to other more consolidated touristic value chains.
\end{abstract}

Keywords: Tourism potential; Ecotourism; Tirimbina Biological Reserve (RBT); Environmental education.

\title{
Introducción
}

El distrito de La Virgen de Sarapiquí, en la provincia de Heredia, Costa Rica, posee una amplia gama de atractivos turísticos para el turista nacional y extranjero. Uno de estos sitios es la RBT, la cual fue declarada "Refugio de Vida Silvestre Privado" en el año 2001, afirmando parte de los esfuerzos del país en conservación y educación ambiental. La Reserva permite el contacto directo con la selva tropical, así como la realización de actividades de aventura en el Río Sarapiquí, según la entrevista realizada.

Dentro de los primeros intentos por caracterizar el ecoturismo en su contexto actual, desde hace un par de décadas para acá, Honey $(2008$, p. 6) lo sustenta como aquellos tipos de viajes por ocio, pero de forma responsable, a áreas naturales que permiten la conservación del medio ambiente y mejoran el bienestar de la población local.

Dentro de esta contextualización también resulta clave reseñar tres aspectos esenciales que componen este tipo de turismo: el primero es que las atracciones deben estar predominantemente basadas en la naturaleza, el segundo que las interacciones de los visitantes con esas atracciones deben centrarse en el aprendizaje o la educación, y por último, la experiencia y la gestión del producto debe seguir los principios y prácticas asociados con la ecología, sostenibilidad sociocultural y económica (Weaver y Lawton, 2007, p. 1170). 
De acuerdo con Fierro Bandala et al. (2013, p. 71), el ecoturismo, es una actividad relativamente reciente, ligada al interés de un sector del turismo que busca acceder a experiencias únicas con la naturaleza, las cuales anteriormente eran exclusivas de los especialistas. Este mismo autor define como actividades ecoturísticas: talleres de educación ambiental, observación de fauna y flora, observación geológica, de fósiles y sideral, safari fotográfico y senderismo interpretativo, participación en programas de conservación e investigación biológica, nadar en pozas naturales, y observación de ecosistemas.

Con base en las definiciones anteriores, se puede afirmar que el ecoturismo es un tipo de turismo que brinda sensaciones únicas y muy agradables al turista con una fuerte base en la entrega de conocimiento y con un manejo sustentable de los atractivos turísticos.

Parte importante del comienzo del ecoturismo se debió a que el desarrollo del turismo, inicialmente y aún en la actualidad, es un proceso que ocupa mayormente el litoral y muy poco las serranías; este desarrollo estereotipado ha tenido como consecuencia una degradación de algunos de los recursos para el turismo, más aun los de orden natural, y es por esta situación que se ha obligado a proponer formas frescas de ejecución del turismo, como lo es el "ecoturismo" (Huerta y Sánchez, 2011, p. 542).

El ecoturismo en América Latina, está emergiendo como un nuevo nicho de mercado y ha sido usado políticamente como bandera en campañas electorales (Flores, 2011 y Coppin, s.f.). El ecoturismo se suele confundir con turismo de observación de la naturaleza; el cual es masivo y sin una ética de conservación, mientras que el ecoturismo debe ser selectivo en el número de visitantes a un área, y su principal fin es la educación ambiental, sensibilizando al turista sobre la dinámica natural (Fierro Bandala et al. 2013, p. 71).

Resulta de gran valor teórico y práctico en el ecoturismo el acercamiento al estudio del ecosistema a través de métodos que integran el enfoque ecológico y geográfico (Hasse, 1986). Parte de lo que desarrolla Morera (2001) en sus estudios con respecto hacia lo que genera el ecoturismo, es que este se basa, teóricamente hablando, en incrementar la demanda por hospedaje y alimentación, promoviendo así que aumente la viabilidad de desarrollo de nuevas empresas relacionadas con estos servicios, y aunque algunas son empresas extranjeras, generan empleo y también existe 
una red de comercio local que indirectamente se ve fortalecida con el impulso de la actividad turística.

La mayoría de estudios asociados a la temática ecoturística plantean que este tipo de turismo genera empleo (Weaver y Lawton, 2007; Honey, 2008; Fennell, 2008 y Guzmán y Juárez, 2013), sin embargo, los resultados comparados con el turismo tradicional, según estudios como el de Morera (2001), no se pueden extrapolar para el caso del ecoturismo, especialmente en el caso del empleo.

La presente investigación indaga sobre la RBT, área natural de Costa Rica que es aún poco explorada, con mucho potencial, pero donde no hay antecedentes que evalúen específicamente el potencial turístico de la zona desde un punto de vista biogeográfico. Es por esto, que se hace un recuento de oportunidades de desarrollo turístico en la zona de Sarapiquí, en un sitio con mucho potencial ecoturístico.

El objetivo del estudio es brindar una idea de los principales potenciales en la zona de Sarapiquí desde el punto de vista ecoturístico, determinando un nivel de potencial con base en un recuento de variables de importancia ecoturística y a partir de la descripción de las características de la RBT.

Asimismo, se indagó sobre las actividades enfocadas en líneas ecoturísticas y la oferta turística presente e interconectada con la reserva, lo cual es importante como insumo para identificar polos o sitios turísticos de interés que presentan un mayor potencial de desarrollo de la mano con la línea ambiental que busca seguir promocionando a Costa Rica como un destino de turismo ecológico importante.

\section{Descripción del Área de Estudio}

El cantón de Sarapiquí se encuentra en la zona norte del país de Costa Rica, ocupando el puesto número 10 de la provincia de Heredia. Sarapiquí cuenta con un área de $2140.54 \mathrm{~km}^{2}$ que lo convierte en uno de los cantones más extensos del país; representando el $4.62 \%$ del territorio nacional y el $82 \%$ de la provincia de Heredia. Dividido en cinco distritos: Puerto Viejo, La Virgen, Horquetas, Llanuras del Gaspar y Cureña (Figura 1).

RBT se encuentra ubicada en La Virgen, colindando con la Reserva Forestal Cordillera Volcánica Central (Figura 2). De esta última, se encuentra en el cantón de Sarapiquí: la Reserva Nacional de Fauna Silvestre 
Mario Arroyo-Solórzano Lincenth Rojas-Prendas

Potencial ecoturístico en Áreas Naturales Protegidas. Caso: Reserva Biológica Tirimbina, Sarapiquí, Provincia de Heredia, Costa Rica.

Barra del Colorado, el Parque Nacional Braulio Carrillo y la Reserva Biológica La Selva, esto según la Municipalidad de Sarapiquí (2012).

Figura 1. Cantón de Sarapiquí con sus respectivos distritos.

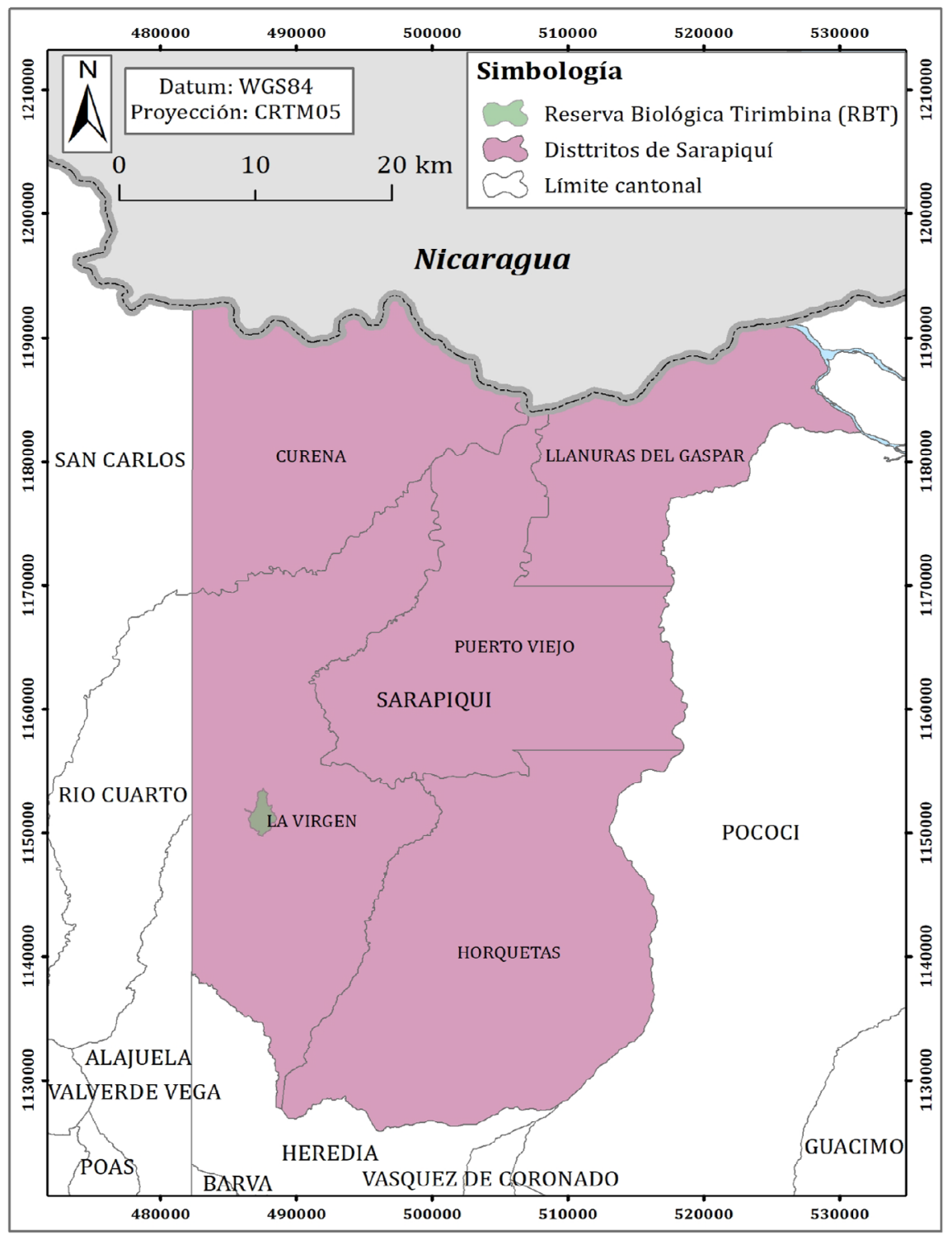

Fuente: elaboración propia. 
Mario Arroyo-Solórzano Lincenth Rojas-Prendas Ecotourism potential in Protected Natural Areas. Case: Tirimbina Biological Reserve (RBT), Sarapiqui, Province of Heredia, Costa Rica.

Figura 2. Ubicación de la RBT.

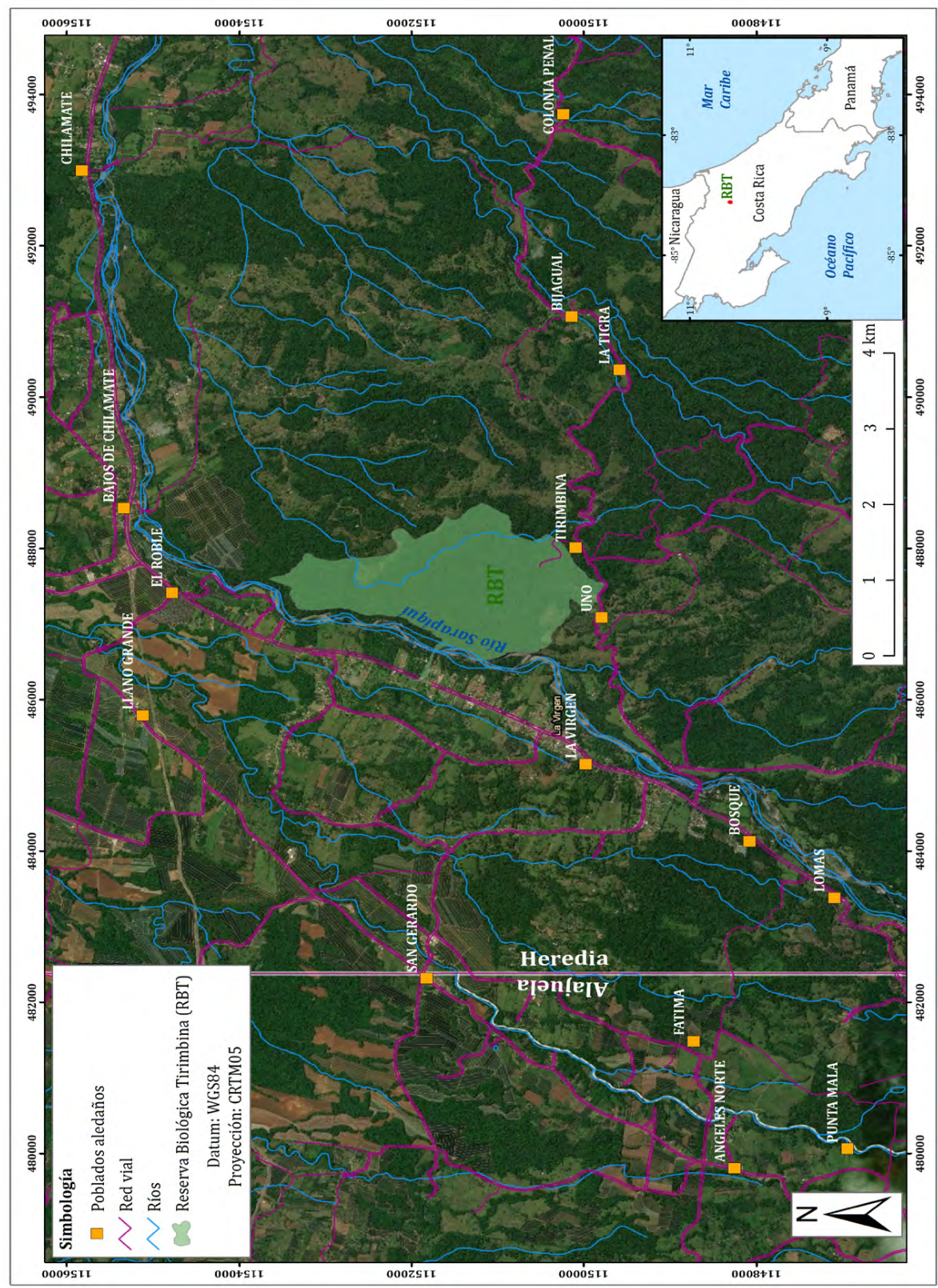

Fuente: Elaboración propia con base en imagen satelital de Google Earth e información de poblados, vías y ríos y del Instituto Geográfico Nacional de Costa Rica (IGN). 
En el distrito de La Virgen, donde se ubica la reserva, se puede encontrar gran cantidad de lugares turísticos para visitar. Uno de éstos, que colinda con la reserva, es el Hotel Sarapiquis Rainforest Lodge (Figura 3), y en los alrededores de La Virgen se encuentran también cabinas y otros alojamientos como Hacienda Pozo Azul.

Figura 3. Piscina del Hotel Sarapiquis Rainforest Lodge.

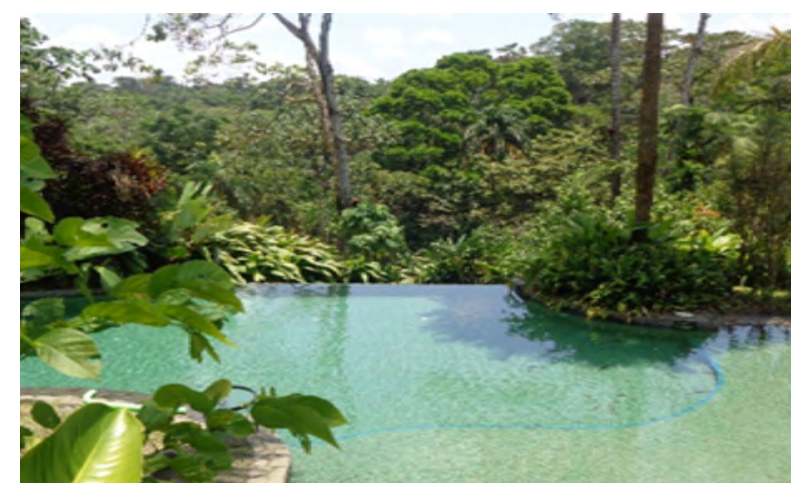

Fuente: Tomada por L. Rojas, 2018.

De acuerdo con la visita realizada al lugar, en lo que respecta a la alimentación, en la zona se presenta gran variedad de $\operatorname{sodas}^{3}$ y restaurantes, así como supermercados y panaderías. En La Virgen de Sarapiquí se pueden realizar diversas actividades de interés turístico como canopy, rafting, rapel, andar a caballo, observar aves y mariposas, nadar en el río Sarapiquí, observar serpientes o simplemente relajarse en algún hotel de la zona.

El cantón de Sarapiquí es rico en potencial turístico, con un exuberante bosque que muestra parte de la biodiversidad de Costa Rica. El acceso a la zona es sencillo, se puede llegar por la Ruta Nacional 126 (Vara Blanca) o Ruta 32 (Zurquí); además en el lugar existe transporte público el cual es accesible, económico y permite trasladarse con facilidad entre los distintos sitios turísticos; esto fue corroborado con la visita realizada al lugar.

\section{Características físico-geográficas}

Sarapiquí tiene características climáticas similares a las de la zona norte de Costa Rica, es decir, un clima típico de la zona tórrida tropical. La precipitación anual es de entre $3500 \mathrm{~mm}$ a $4000 \mathrm{~mm}$; se encuentra una 
época lluviosa marcada de octubre a enero y de mayo a agosto, disminuyendo la cantidad de lluvias de febrero a abril. El viento que suele ser predominante en la zona es suave, la temperatura media oscila entre los $20^{\circ} \mathrm{C}$ a $30^{\circ} \mathrm{C}$, que aumenta en las zonas bajas y disminuye en las proximidades de la Cordillera Volcánica Central. La humedad relativa va de $80 \%$ a $90 \%$. Según las clasificaciones climáticas desarrolladas por Thornthwaite (1948) y Herrera (1985), los climas de Sarapiquí se pueden resumir en:

1. Clima muy húmedo, muy caliente

2. Clima muy húmedo, caliente

3. Clima excesivamente húmedo, templado

4. Clima muy húmedo, templado

5. Clima muy húmedo, frío

6. Clima excesivamente húmedo, frío

En el cantón existen 8 zonas de vida de Holdridge (1967, p. 19-29); del cual se deduce que la más representativa en la región es "Bosque muy húmedo tropical”, abarcando el $73 \%$ del área total (Vega, 2014, p. 21). El territorio está localizado en la subvertiente norte de la Vertiente Caribe. A esta subvertiente le pertenecen las cuencas de los ríos Sarapiquí, Sucio, Chirripó y Cureña. Con base en la visita realizada al lugar, el Río Sarapiquí es de gran extensión y tiene un gran potencial turístico debido a su belleza escénica y a sus rápidos que son aprovechados en actividades como el rafting (Figura 4) y en el curso inferior o cuenca baja para practicar la pesca, y la navegación deportiva y turística.

Figura 4. Rafting en el río Sarapiquí, vista desde el puente colgante de Tirimbina.

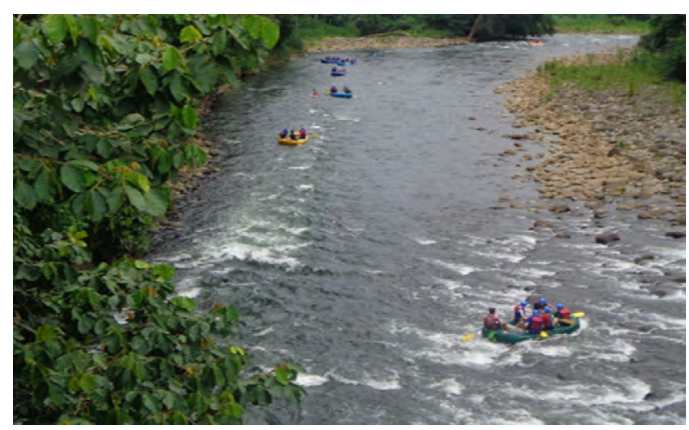

Fuente: Tomada por L. Rojas, 2019. 
La mayoría de los suelos en el cantón de Sarapiquí son del orden Inceptisol (44\% del área total), seguido por los Ultisoles con 39\% del área total. Geomorfológicamente, Vega (2014, p. 29); menciona que Sarapiquí presenta 2 unidades principales; la de sedimentación aluvial y la de origen volcánico.

La de sedimentación aluvial está dividida en 5 subunidades que son:

1. Llanura Aluvial de San Carlos y el Caribe.

2. Llanuras Bajas Recientes.

3. Pantano Permanente o Temporal.

4. Abanico Aluvial del Río Chirripó y Sucio.

5. Abanico Aluvial de los ríos Cuarto y Sarapiquí.

Las formas de origen volcánico se subdividen en 2 unidades que son:

1. La subunidad Volcán Barva.

2. La subunidad Cerros y Colinas del Vulcanismo de Intragraben.

\section{Historia y Formación de la Reserva}

Tirimbina es un Refugio Nacional de Vida Silvestre Privado, cuenta con 345 hectáreas de bosque, de las cuales 335 ha son bajo pago por servicios ambientales (PSA). Cuenta con $9 \mathrm{~km}$ de senderos para turismo y educación. La Reserva es una organización sin fines de lucro y se centra en un modelo de investigación, educación y ecoturismo para la conservación.

Debido a esto, en Tirimbina se busca proteger la excepcional biodiversidad de la zona mediante la educación ambiental y la investigación científica que promueve la conservación. La Reserva pretende ser un modelo de conservación sólido, autosuficiente y armonioso con el impacto local, nacional e internacional según la información recopilada en el sitio.

Como se detalla en el apartado metodológico, se realizó una entrevista a parte del personal actual de la RBT, donde esta persona detalló que la historia de Tirimbina se remonta a 1960 con la llegada del estadounidense Robert Hunter al cantón de Sarapiquí, el cual investigaba cultivos novedosos en esta zona y adquiere terrenos con bosque, entre ellos Tirimbina. Hunter contactó a Allen Young, un investigador del Museo de Milwaukee quien publicó más de 100 investigaciones sobre insectos y el cacao.

En 1986 el Museo de Milwaukee toma el bosque de Tirimbina como base para diseñar en los Estados Unidos una exhibición sobre el bosque tropical. Hunter en el año de 1989 inicia acuerdos con el CATIE (Centro 
Agronómico Tropical de Investigación y Enseñanza) para realizar más investigaciones en el bosque. Desde ese momento muchos proyectos de investigación se han realizado en esta zona.

En 1995, Hunter vende la propiedad, siendo la compra patrocinada por el Centro Natural Riveredge y el Museo Público de Milwaukee, y es de este acuerdo donde nace Tirimbina. En 1999, Tirimbina inicia sus operaciones y programas de Educación Ambiental y Riveredge deja el patrocinio.

Tirimbina es declarada "Refugio de Vida Silvestre Privado" afirmando sus esfuerzos de conservación y educación en el año 2001. En el 2005, el Museo de Milwaukee sale del manejo y Tirimbina asume el 100\% de las operaciones. En el 2009,se crea la Asociación Tirimbina para la Conservación, Investigación y Educación (ATCIE); una asociación sin fines de lucro y la cual hasta hoy día es la propietaria del terreno de Tirimbina.

\section{Marco Metodológico}

Recopilación bibliográfica

Se recopiló información de publicaciones, informes de proyectos de investigación y gubernamentales y la cartografía existente de la zona sobre geomorfología, geología, climas, hidrología, cobertura y uso del suelo y biodiversidad; así como información geológica y edáfica. Se evaluó la parte investigativa y educacional, así como de ecoturismo en general de la Reserva y principalmente se obtuvo información primaria a través de la visita al campo y la realización de una entrevista detallada. Todos los datos se indagaron, recolectaron y para finalizar se analizaron, obteniendo lo más relevante de la reserva para propósitos de esta investigación.

\section{Entrevista realizada}

La información obtenida proviene principalmente de un importante trabajo de campo, el cual permitió un mayor conocimiento del área de interés. En este proceso una de las principales fuentes de información fue la entrevista realizada a una de las personas encargadas del área de investigación de la Reserva, Mariela García (García, 2019), la cual permitió obtener información importante y de primera mano para la investigación, junto con las visitas para el recorrido y participación en tours dentro de la RBT. La entrevista básicamente consistió en un proceso de indagación donde se realizaron consultas generales con preguntas abiertas y la persona 
comentaba al respecto. En este sentido se buscaba conocer más en detalle la evolución histórica de la RBT, los programas de educación que implementan, los servicios generales que se brindan y las oportunidades de tours que ofertan o manejan.

\section{Evaluación del Potencial Ecoturístico}

Se definieron modelos teóricos óptimos para la realización de actividades ecoturísticas y de aventura, basados en atributos biofísicos del paisaje. Estos modelos se modificaron de los propuestos por Acosta (2008). Las variables se evaluaron cualitativamente, con una propuesta de clasificación, cruzándose con calidades evaluativas de lo que ofrece la RBT en estas variables, a modo de matriz.

La elección de la metodología se basó en la posibilidad de evaluación de los parámetros o variables en función de las características de la RBT y de la factibilidad de verificación de estas con base en criterios de observación de campo y procesos de indagación al personal de la Reserva, así como de acuerdo con el contexto donde se ubica la misma en el cantón de Sarapiquí. Se propone una metodología práctica y centrada en evaluar aspectos naturales de interés ecoturístico que se compara brevemente en la discusión con otras que siguen líneas similares como es el caso de la implementada por Huerta y Sánchez (2011, p.541-560).

Las variables evaluadas fueron seis: senderismo, observación de aves, observación de flora y fauna silvestre, diversidad de paisajes naturales, actividades de aventura y por último la seguridad y accesibilidad. A estas se le asignaron claves o pesos de potencial, de acuerdo con su correspondencia, entre Alto (3), Medio (2) y Bajo (1).

Los aspectos contra los que se evaluaron las variables fueron cinco: complejidad, riqueza natural, singularidad, número de especies y cuerpos de agua. Metodologías alternativas proponen otras múltiples variables (Huerta y Sánchez, 2011), pero en este caso se tomaron estas variables mencionadas como las más relevantes, tomando en cuenta la practicidad de su evaluación, el ser un único sitio especifico por evaluar y basados en una evaluación meramente natural del potencial ecoturístico, no social ni económica en detalle.

El proceso de verificación de estos aspectos contempló la visita en cinco oportunidades a la RBT durante los meses de enero, marzo, mayo, julio y setiembre, donde se recorrieron los senderos disponibles y se 
realizaron parte de los tours que ofrece la Reserva. La Tabla 1 muestra los aspectos que se tomaron como referencia para establecer los pesos, contemplando los procesos de observación en cada época del año.

Tabla 1. Aspectos para evaluar las variables del potencial ecoturístico en el área de interés.

\begin{tabular}{|c|c|c|c|}
\hline $\begin{array}{l}\text { Aspectos de } \\
\text { evaluación }\end{array}$ & Peso 3 (Alta) & Peso 2 (Media) & Peso 1 (Baja) \\
\hline Complejidad & $\begin{array}{c}\text { Se presenta gran di- } \\
\text { versidad y variedad } \\
\text { de trayectos, activi- } \\
\text { dades y especies en } \\
\text { todos los recorridos } \\
\text { del lugar, la mayoría } \\
\text { accesibles a todas las } \\
\text { personas. }\end{array}$ & $\begin{array}{c}\text { Se presenta gran di- } \\
\text { versidad y variedad de } \\
\text { trayectos, actividades } \\
\text { y especies en más de la } \\
\text { mitad de los recorridos } \\
\text { del lugar, accesibles a } \\
\text { todas las personas el } 50 \% \\
\text { de estos. }\end{array}$ & $\begin{array}{c}\text { Se presenta gran diversi- } \\
\text { dad y variedad de trayec- } \\
\text { tos, actividades y especies } \\
\text { en menos de la mitad de } \\
\text { los recorridos del lugar. } \\
\text { La mayoría de los sitios } \\
\text { no son accesibles a todas } \\
\text { las personas. }\end{array}$ \\
\hline $\begin{array}{l}\text { Riqueza } \\
\text { natural }\end{array}$ & $\begin{array}{l}\text { Cualquier sitio } \\
\text { presenta una gran } \\
\text { biodiversidad y la } \\
\text { mayoría son acce- } \\
\text { sibles a todas las } \\
\text { personas. }\end{array}$ & $\begin{array}{c}\text { Al menos el } 50 \% \text { del } \\
\text { lugar presenta sitios con } \\
\text { abundante biodiversidad } \\
\text { y son accesibles a todas } \\
\text { las personas. }\end{array}$ & $\begin{array}{l}\text { Menos del } 50 \% \text { del lugar } \\
\text { presenta abundante bio- } \\
\text { diversidad y accesible a } \\
\text { todas las personas. }\end{array}$ \\
\hline Singularidad & $\begin{array}{c}\text { Características } \\
\text { únicas en las zonas } \\
\text { aledañas y muy } \\
\text { pocos sitios similares } \\
\text { a nivel país }\end{array}$ & $\begin{array}{c}\text { Características únicas en } \\
\text { las zonas aledañas y con } \\
\text { zonas similares a nivel } \\
\text { país }\end{array}$ & $\begin{array}{l}\text { Características similares } \\
\text { a la zona en general y con } \\
\text { muchas zonas del país } \\
\text { parecidas }\end{array}$ \\
\hline $\begin{array}{l}\text { Número de } \\
\text { especies }\end{array}$ & $\begin{array}{l}\text { La cantidad de es- } \\
\text { pecies permite su } \\
\text { fácil observación en } \\
\text { cualquier sitio y la } \\
\text { mayoría del año }\end{array}$ & $\begin{array}{l}\text { La cantidad de especies } \\
\text { permite su fácil obser- } \\
\text { vación en la mayoría del } \\
\text { sitio y solo en ciertas } \\
\text { épocas del año }\end{array}$ & $\begin{array}{l}\text { La cantidad de especies } \\
\text { no es suficiente para su } \\
\text { fácil observación en la } \\
\text { mayoría del sitio y solo se } \\
\text { aprecian en ciertas épocas } \\
\text { del año }\end{array}$ \\
\hline $\begin{array}{l}\text { Cuerpos de } \\
\text { agua }\end{array}$ & $\begin{array}{l}\text { Los cuerpos de agua } \\
\text { son de grandes ex- } \\
\text { tensiones y están en } \\
\text { más del } 25 \% \text { del área }\end{array}$ & $\begin{array}{l}\text { Los cuerpos de agua son } \\
\text { de moderada extensión } \\
\text { y están en más del } 10 \% \\
\text { del área }\end{array}$ & $\begin{array}{l}\text { Los cuerpos de agua son } \\
\text { escasos o nulos }\end{array}$ \\
\hline
\end{tabular}

Fuente: Elaboración propia, basado en criterios de Acosta (2008, p.109).

Estas relaciones permitirían tener una visualización general del potencial ecoturístico de la RBT, principalmente con la visita en distintas 
épocas del año. Con base en este cruce de variables y la ponderación propuesta con pesos de uno a tres, la suma máxima posible sería de 72 . De acuerdo con esto, se propone una clasificación final del potencial ecoturístico del sitio en cuatro posibles clases: baja, moderada, alta y muy alta, de acuerdo con la suma de los pesos para cada variable evaluada (Tabla 2).

Para el manejo general de los datos y presentación de los gráficos, figuras y mapas se utilizaron los programas Microsoft Office Excel, ArcMap 10.5, Adobe Illustrator CS6 y Google Earth. En la sección de resultados se muestra la matriz con el cruce de variables y su clasificación, según lo evaluado en la RBT.

Tabla 2. Propuesta de clasificación del potencial ecoturístico con base en la sumatoria de las variables evaluadas.

\begin{tabular}{|c|c|}
\hline Potencial ecoturístico & Sumatoria de las variables \\
\hline Bajo & $0-18$ \\
\hline Moderado & $19-36$ \\
\hline Alto & $37-54$ \\
\hline Muy alto & $55-72$ \\
\hline
\end{tabular}

Fuente: Elaboración propia.

\section{Resultados}

- Generalidades de la RBT

En Tirimbina habita un $25 \%$ de los anfibios observables en Costa Rica, un $43 \%$ de los mamíferos, $13 \%$ de la flora del país, 401 especies con agallas (excrecencia redonda que se forma en ciertos árboles y plantas a partir de la picadura de algunos insectos y de las infecciones producidas por microorganismos), 24\% de los reptiles observables en Costa Rica, un $41 \%$ de las aves observables, $10 \%$ de especies de mariposas del país y 29 especies de los dulceacuícolas (organismos que viven en agua dulce) y que representa el $22 \%$ de las observables en Costa Rica; esto con base en la entrevista realizada y de acuerdo también con Durán (s.f.).

La Reserva actualmente cuenta con cinco proyectos de investigación los cuales son: Fenología de palmas, Monitoreo del bosque tropical lluvioso, Diversidad y reproducción de aves (Figura 5), Proyecto Lepidóptera: 
y Monitoreo de murciélagos. Tirimbina también presenta como característica llamativa, que es hogar del murciélago blanco (Ectophylla alba), especie que se encuentra únicamente en la vertiente caribeña de Costa Rica y países como Honduras, Nicaragua y el noroeste de Panamá.

Este murciélago utiliza plantas como las heliconias (muy abundantes en la Reserva) para construir unas "tiendas" (Figura 6) donde duermen y se protegen durante el día. No utiliza cuevas ni arboles huecos como otras especies de murciélagos. Su pelaje blanco le sirve para reflejar la luz verde que se filtra a través de la hoja de la platanilla; así se esconden agrupados, entre las hojas, y se protegen de depredadores que pueden atacar desde el suelo.

Figura 5. Manacus candei (manaquín cuello blanco), especie muy común que se puede apreciar en la zona.

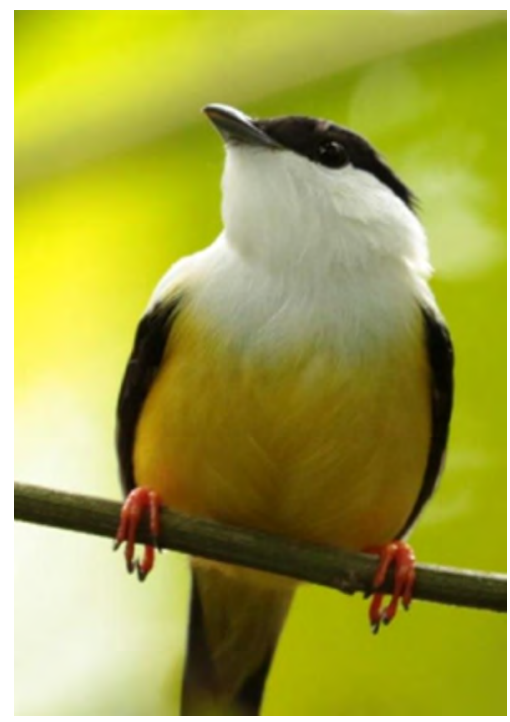

Fuente: Tomada de García (2019).

La RBT cuenta con un programa de educación ambiental desde 1999 el cual ha mejorado en el tiempo y en la actualidad es conocido como "Aula en el bosque". Con esta iniciativa se busca que los escolares estudien los contenidos de ciencias relacionados con ambiente y desarrollo sostenible de manera vivencial, facilitándoles la integración de conocimientos por 
Figura 6. Ectophylla alba (murciélago blanco) en una "tienda".

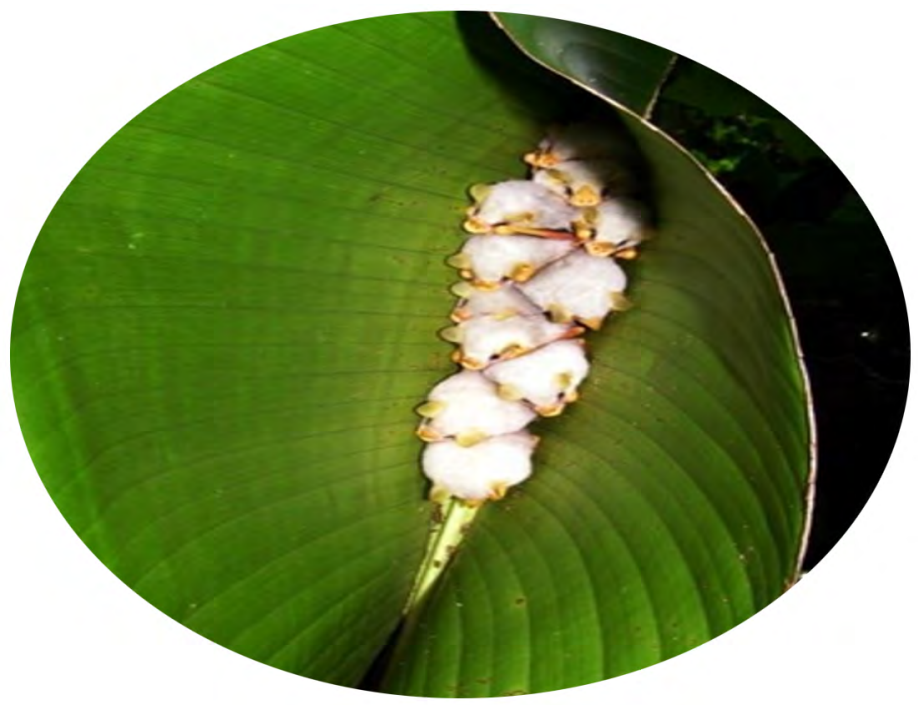

Fuente: Tomada de García (2019).

medio de trabajo de campo, en contacto directo con el objeto de estudio, reseñado así por la persona entrevistada.

En cuanto a programas actuales de ecoturismo que se ofrecen al turista en la Reserva, algunos de los principales tienen que ver con tours guiados de caminata nocturna para visualizar animales, otros diurnos para visualización de aves, tours de chocolate donde se enseña sobre el procesamiento del cacao y las preparaciones de chocolates, tours de historia natural con explicación de guías locales, tour de murciélagos el cual es de los más llamativos por la singularidad de la especie reseñada en la Figura 6 y por último los tours libres o de caminata autoguiada. Es importante reseñar que en la Reserva se cuenta con servicio de hospedaje y restaurante (Reserva Biológica Tirimbina, s.f.).

Siguiendo la línea de educación e investigación que reseñan los múltiples autores en cuanto a la conceptualización del ecoturismo, la RBT se presenta como un sitio turístico que responde bastante a la tendencia y conceptualización de actividades ecoturísticas, con programas educativos, tours basados en brindar al turista conocimiento sobre el sitio y con la 
mentalidad de preservar la riqueza en cuanto a biodiversidad presente. Sin embargo, estos aspectos parecen carecer de un enfoque de atracción que se oriente más hacia la promoción de la visita al sitio en mayor medida. Son aspectos poco dados a conocer a nivel nacional, con débiles encadenamientos actuales a otros atractivos de la zona o zonas aledañas.

- Evaluación de potencial para el Ecoturismo

Se evaluó el potencial de las unidades de paisaje para el desarrollo de seis variables ecoturísticas y de aventura: senderismo, observación de aves, observación de flora y fauna silvestre, diversidad de paisajes naturales, actividades de aventura, seguridad y accesibilidad para todas las personas.

La Tabla 3 muestra la matriz de la evaluación y ponderación de pesos hecha para la RBT, lo cual es basado en los criterios expuestos en la Tabla 1 y determinado de acuerdo con en el proceso de inspección y evaluación con base en la observación por medio de las visitas a la Reserva. En el Grafico 1 se aprecian las variables que más sobresalen, siendo la observación de flora y fauna silvestre la más sobresaliente.

La suma de los pesos en las variables fue de 46. Esto categoriza a la RBT como un lugar con Alto potencial ecoturístico, según la clasificación dada en la Tabla 2. Se obtuvo un alto potencial en complejidad, riqueza natural y número de especies en la observación de la flora y fauna, siendo esta variable la de mayor potencial en Tirimbina, ya que es sumamente factible poder observar flora y fauna en el lugar y se encuentra una gran variedad de especies en cualquier época del año.

En la única variable que se encontró un alto potencial en singularidad fue en la diversidad de paisajes naturales, debido a que Tirimbina tiene una característica única y que pocos sitos en el país comparten: la presencia de un río como el Sarapiquí que se encuentra en medio de la reserva y la atraviesa, además que en algunos lugares es fácil observar cuerpos de agua que hacen del lugar un paraje peculiar. En la mayoría de las épocas del año en que se visitó se corroboró esto, a excepción de marzo donde se tenía un menor nivel de agua.

A la observación de aves se le asignó un peso medio, puesto que, aunque existe gran variedad de aves para observar, algunas veces se dificulta el avistamiento de estas, o simplemente no en todos los senderos de la reserva se logran apreciar, ni en todas las épocas del año. Las actividades 
de aventura son escasas, siendo la única el senderismo que predomina en Tirimbina, sin embargo, la riqueza natural que se encuentra al realizar esta actividad es muy alta, y también los dos puentes colgantes que se encuentran durante el recorrido le dan un valor agregado a este tipo de actividades.

El peso más bajo es asignado a la seguridad y accesibilidad para todos, debido a que a pesar de la zona es en general muy segura, en cuanto a la accesibilidad para personas con alguna discapacidad o adultos mayores, el acondicionamiento de la Reserva y sus senderos es casi nulo.

A los únicos lugares que se tiene acceso para estas personas es al jardín y a los senderos que se encuentran al entrar a Tirimbina, restaurante y habitaciones, esto según la observación de campo realizada. La realización de tours para estas personas es casi imposible, siendo esto un punto negativo para el lugar, donde el tour de murciélagos es el único que presentaría esa accesibilidad.

Tabla 3. Matriz de evaluación del potencial ecoturístico en Tirimbina con los respectivos pesos (Tabla 1).

\begin{tabular}{|c|c|c|c|c|c|}
\hline $\begin{array}{c}\text { Variables por } \\
\text { evaluar }\end{array}$ & $\begin{array}{c}\text { Compleji- } \\
\text { dad }\end{array}$ & $\begin{array}{c}\text { Riqueza } \\
\text { natural }\end{array}$ & Singularidad & $\begin{array}{c}\text { Número de } \\
\text { especies }\end{array}$ & $\begin{array}{c}\text { Cuer- } \\
\text { pos de } \\
\text { agua }\end{array}$ \\
\hline $\begin{array}{c}\text { Senderismo } \\
\begin{array}{c}\text { Observación de } \\
\text { aves }\end{array}\end{array}$ & Peso 2 & Peso 3 2 & Peso 2 & NA & Peso 2 \\
\hline $\begin{array}{c}\text { Observación de } \\
\text { flora y fauna } \\
\text { silvestre }\end{array}$ & Peso 3 & Peso 3 & Peso 2 & Peso 3 & NA \\
\hline $\begin{array}{c}\text { Diversidad } \\
\text { de paisajes } \\
\text { naturales }\end{array}$ & Peso 2 & Peso 2 & Peso 3 & NA & Peso 2 \\
\hline $\begin{array}{c}\text { Actividades de } \\
\text { aventura }\end{array}$ & Peso 1 & Peso 3 & Peso 1 & NA & NA \\
\hline $\begin{array}{c}\text { Seguridad } \\
\text { accesibilidad } \\
\text { para todas las } \\
\text { personas }\end{array}$ & Peso 1 & Peso 1 & Peso 1 & NA & NA \\
\hline
\end{tabular}

NA: No aplica. Fuente: Elaboración propia. 
Gráfico 1. Pesos asignados por variables.

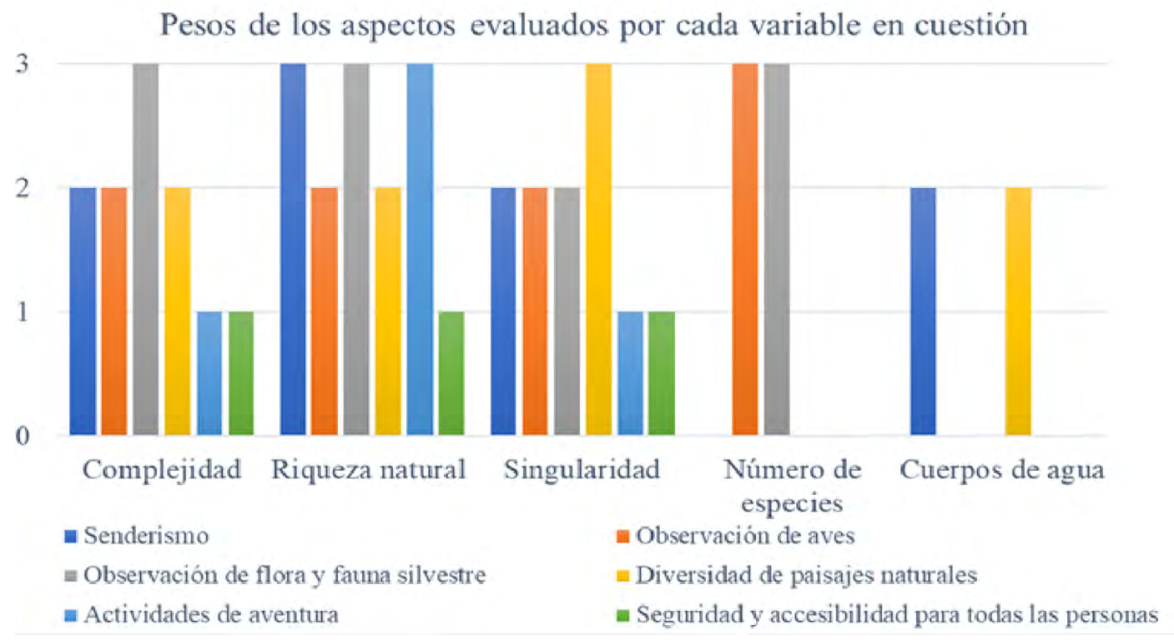

Fuente: Elaboración propia.

\section{Discusión}

Tirimbina es un sitio con un gran potencial turístico en Sarapiquí. Las características peculiares que presenta la Reserva la convierten en uno de los mejores lugares para el turismo en el distrito de la Virgen. Los residentes de la zona de La Virgen de Sarapiquí visitan constantemente el lugar, ya que es un espacio lleno de tranquilidad, biodiversidad y exuberante belleza natural. La Reserva se encuentra bien organizada y ofrece variedad de servicios para el turista, enfocándose en la conservación del ambiente, educación y el ecoturismo, brindando al turista una cantidad de recorridos muy completos para el disfrute del lugar.

La RBT obtiene a nivel de potencial ecoturístico evaluado, un término Alto, siendo el senderismo la riqueza natural que se encuentra con mejor ponderación a nivel general de los aspectos evaluados. En todos los senderos se puede apreciar una exuberante naturaleza, están rodeados de bosque, se puede valorar con facilidad la flora y fauna del lugar, así como los dos puentes colgantes que hay que atravesar y uno de ellos pasa sobre el río Sarapiquí, presentándose este como un río limpio, fresco y de vital 
importancia para la zona, ya que en él se realizan distintas actividades para el turismo.

La observación de la flora y fauna silvestre presenta un papel bastante importante en el lugar, siendo de las variables con mayor potencial. En Tirimbina es fácil observar monos, iguanas, osos perezosos, murciélagos y gran cantidad de especies de aves.

Un mamífero muy llamativo que se encuentra en la Reserva es el Serafín de platanar (Figura 7), una especie de oso hormiguero que es fácil de observar en el lugar, pero es un animal muy poco visto en el país y casi no conocido por las personas. La flora también es abundante en toda la reserva y estos aspectos hacen de estas variables de las de mayor potencial.

La observación de aves en el lugar fue categorizada con un potencial medio, ya que no se asegura apreciar en todos los senderos especies de aves, pero la diversidad que se encuentra es alta y un ejemplo es el avistamiento del martín pescador vientrirrufo (Chloroceryle inda), una de las varias especies más peculiares que se puede apreciar en la Reserva. En Tirimbina la diversidad de paisajes no es muy distinta a lo que se puede encontrar en otros lugares del país, pero la presencia del Río Sarapiquí le da un atractivo importante desde el punto de vista paisajístico.

Figura 7. Cyclopes didactylus (serafín de platanar).

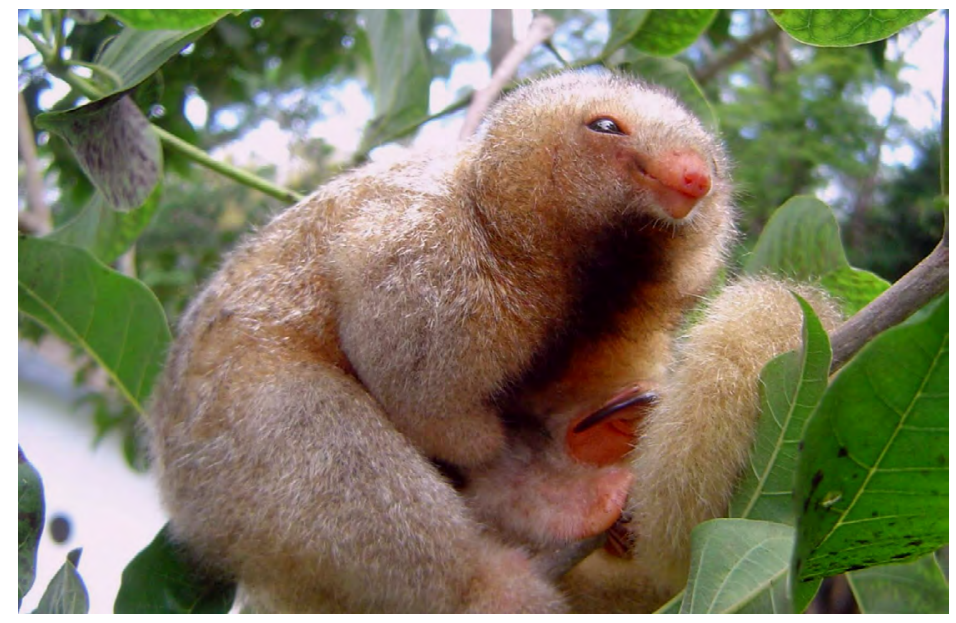

Fuente: Tomada de El Norte Hoy (2019). 
Las actividades de aventura son escasas, siendo una el senderismo, pero el nivel de riqueza natural que se aprecia mientras se realizan los recorridos es basto e impresionante. Las dificultades en cuanto a la accesibilidad para personas con alguna discapacidad o adultos mayores en cuanto realizar los tours es un aspecto que limita el sitio, con escasos o nulos senderos para personas con alguna discapacidad o adultos mayores.

Por ejemplo, para adentrarse en el bosque y realizar parte de los recorridos, hay que atravesar un puente colgante que pasa por encima del Río Sarapiquí y es el único medio para poder trasladarse hasta el otro lado de la reserva. Dicho puente colgante no es apto para discapacitados o adultos mayores, siendo los senderos que se encuentran a la entrada de la reserva, el jardín, habitaciones y restaurante, los únicos lugares a los que tienen acceso estas personas.

Al comparar los aspectos evaluados y los resultados obtenidos de la metodología propuesta, con otros estudios que siguen líneas similares como es el caso del estudio o categorización de Huerta y Sánchez (2011, p. 541-560), donde hay diferencias puntuales y de gran importancia en cuento a la escala y el alcance en la presente investigación, con respecto a dichos estudios. Esta investigación propone una categorización y ponderación de aspectos meramente de tipo natural para la evaluación especifica de sitios con potencial ecoturístico en zonas tropicales, con características climáticas similares a la RBT.

El estudio de Morera (2001) contempla una indagación en la zona Sur de Costa Rica, donde se relacionan más en detalle las actividades turísticas, desde el punto de vista de oportunidades económicas y de empleo o de servicios en la zona, con atractivos ecoturísticos. En la presente investigación se busca principalmente categorizar el potencial ecoturístico de la RBT y se indaga en evaluar si la Reserva cumple con los principios ecoturísticos mundiales.

La principal diferencia con respecto al estudio de Huerta y Sánchez (2011, 541-560), p; es que en esa investigación se contempló una evaluación más regional, al evaluarse un corredor ecoturístico comunitario desde puntos de vista naturales, sociales y económicos en detalle, es decir, en esa investigación los autores ponderan o categorizan distintos sitios dentro de un corredor natural ya previamente determinado como ecoturístico. En la presente investigación se busca proveer un tipo de categorización 
ecoturística para la RBT, con principios similares al estudio de Huerta y Sánchez (2011), pero para un sitio especifico que se está evaluando como posible lugar ecoturísticamente aprovechable y categorizando solo desde el punto de vista natural.

La existencia de múltiples metodologías y orientaciones en la evaluación del potencial turístico y en este caso con enfoque ecológico (ecoturismo) lo que denotan es que hay diversas maneras que pueden ser válidas para interpretar las características de un sitio o zona turística, según los objetivos o lo que se quiere evaluar en cada investigación.

La metodología aplicada, a modo general, resultó ser bastante concordante con los aspectos de tipo natural evaluados los otros estudios reseñados y donde las variables naturales suelen basarse en aspectos de biodiversidad y características únicas o particulares que resaltan a los sitios naturales que se evalúan. Se podría pensar en la realización futura de una integración con la metodología de Huerta y Sánchez (2011) para la RBT, donde se categorice y ponderen las características sociales y económicas asociadas con la Reserva.

Con base en lo indagado, el lugar tiene mucho que ofrecer al turismo desde sus características naturales, categorizándose como de alto potencial, sin embargo, también se identificó que la Reserva podría aprovechar aún más su potencial ecoturístico. Se debe de invertir principalmente en mejorar y crear nuevos senderos, así como reinventar algunos recorridos. La RBT además de ser un gran potenciador del turismo a la zona de La Virgen de Sarapiquí, es una gran fuente de empleo para los habitantes que podría potenciarse.

Según el Índice de Desarrollo Humano cantonal (IDHc), Sarapiquí fue el único cantón de la provincia de Heredia con un IDHc por debajo del promedio nacional para el 2011 y en general toda la Región Huetar Norte, a la cual pertenece Sarapiquí, presenta un nivel de pobreza del 27,5\% (INEC, 2020 y PNUD, 2011).

Es por lo anterior que, el sector de servicios de la mano de principal actividad productiva del país como lo es el turismo, podría ser un mayor impulso para ayudar a la población de la zona. En el lugar el emprendimiento y los encargados del sector servicios son principalmente vecinos de la zona, lo cual ayuda al desarrollo de esta en ese sector productico. 
Al evaluarse los criterios señalados como base para la práctica ecoturística (riqueza natural, educación/aprendizaje y sostenibilidad), parece ser que la RBT cumple con la línea ecoturística. Sin embargo, dentro de estos aspectos, la parte de sostenibilidad debe ser entendida con especial cautela, ya que, si bien se busca la sostenibilidad ambiental y practicas amigables con el ambiente, este no es el único aspecto de sostenibilidad que denotan las teorías ecoturísticas, sino que también contemplan una sostenibilidad social y económica con base y/o soportada en parte, por la actividad ecoturística (Weaver y Lawton, 2007).

Es así como cuando se inspeccionan aspectos como lo señalados en los párrafos anteriores, se puede deducir que la zona de Sarapiquí no está viendo beneficios en la medida deseada para cumplir con las líneas ecoturísticas ideales, o lo que podría aportar el ecoturismo a esta región.

La RBT ofrece gran variedad de servicios para el turista, tanto extranjero como nacional, enfocándose en un turismo de conservación y educativo, siguiendo una línea ecoturística según los principios expuestos en la conceptualización del ecoturismo a nivel mundial. La Reserva necesita mayor inversión y se debe fortalecer, esto con base en lo observable durante el trabajo de campo y también basados en la necesidad poblacional, según lo indagado y en el impulso que esta actividad puede generar para la economía de la zona.

\section{Conclusiones}

La RBT se centra en la conservación del ambiente, la educación y el turismo natural. La Reserva ofrece diferentes actividades para el turista extranjero, así como el nacional, cuenta con un gran potencial turístico, sin embargo, muchos de los costarricenses desconocen de su existencia.

Sarapiquí ofrece gran cantidad de atractivos para el turista y también cuenta con variados servicios gastronómicos y de hospedaje. El Río Sarapiquí es de suma importancia para la zona y es apto para el esparcimiento y la recreación. El río no se encuentra contaminado y muchos grupos turísticos sacan gran provecho a sus rápidos.

Tirimbina es un lugar con una selva tropical exuberante, cuenta con gran cantidad de flora y fauna de Costa Rica y es un lugar muy confortable y de mucha belleza para visitar. Los paisajes naturales y las características 
únicas que tiene lo convierten en una de las mejores opciones para realizar turismo en la zona de Sarapiquí.

Se ha propuesto una metodología para la evaluación del potencial ecoturístico natural, donde la RBT se ha catalogado como de alto potencial, resaltado, entre otras cosas, aspectos como la facilidad de observación de gran cantidad de flora y fauna, paisajes únicos y una amplia variedad de recorridos para el senderismo.

Se sugiere para futuras investigaciones evaluar y categorizar con mayor detalle los aspectos de sostenibilidad social y económica de la zona de Sarapiquí, para contemplar el concepto de sostenibilidad ecoturista integralmente (natural, social y económica), esto de la mano con el equilibrio y la preservación ambiental.

La RBT, de acuerdo con este análisis, podría convertirse en una de las opciones favoritas para el turista, tanto extranjero como nacional y atraer mayor inversión a la zona de Sarapiquí. El lugar a pesar de tener un gran potencial ecoturístico cuenta con algunas debilidades, y la mayor de ellas es la accesibilidad para las personas adultas mayores o personas con discapacidad. Los senderos especializados para realizar la mayoría de los recorridos no cuentan con accesibilidad para estas personas.

La Reserva promueve la protección ambiental, la educación y puede convertirse en un gran potenciador del turismo en la zona; conformándose una cadena turística donde otras empresas dedicadas a brindar servicios relacionados con el turismo, como servicios gastronómicos y de hospedaje, resulten también beneficiadas.

Además, en una visión turística integrada, Tirimbina podría ligarse con otros atractivos turísticos relativamente cercanos, como, por ejemplo, el Volcán Poas, la Laguna Hule, Cataratas de Bajos del Toro o la zona del Volcán Arenal en La Fortuna de San Carlos y sus sitios aledaños, convirtiéndose en parte de los atractivos o sitios por visitar, ligándose a ese tipo de encadenamientos turísticos más consolidados y dando pie a futuras evaluaciones integrales y categorizaciones de sitios en corredores ecoturísticos a nivel nacional.

\section{Referencias}

Acosta Villegas, M. (2008). Evaluación del potencial ecoturístico de un sector de la región Sierra-Costa de Michoacán. (Tesis inédita de 
Mario Arroyo-Solórzano Lincenth Rojas-Prendas Ecotourism potential in Protected Natural Areas. Case: Tirimbina Biological Reserve (RBT), Sarapiquí, Province of Heredia, Costa Rica.

Maestra). Universidad Nacional Autónoma de México, Morelia, México. 109 pp.

Coppin. L (s.f.). Ecoturismo y América Latina: una aproximación al tema.

Tourism as Economic Activity: OTS in Costa Rica, FPEI Working Paper $\mathrm{N}^{\circ} 33.8 \mathrm{pp}$.

Durán. F. (s.f.). Lugar de publicación: ECOBIOSIS. Disponible en: http:// ecobiosis.museocostarica.go.cr/ecosistemas/index.aspx

Fennell, D. (2008). Ecotourism. $3^{\text {ra }}$ ed. London y New York: Ed. Routledge and Taylor \& Francis.

Fierro Bandala, E.; Lagunas Vázques, M. y Ortega Rubio, A.; (2013). El ecoturismo y el turismo de naturaleza como alternativa de desarrollo rural en la REBIOSLA. En: Lagunas Vázques M.; Beltrán Morales, L. y Ortega Rubio, A; (2013). Diagnóstico y análisis de los aspectos sociales y económicos en la reserva de la biosfera Sierra La Laguna, Baja California Sur, México. Centro de Investigaciones Biológicas del Noroeste S.C. La Paz, B.C.S., México. 340 pp.

Flores, Á. (2011). "Zonificación funcional ecoturística de la zona costera de Michoacán, México a escala 1:250 000”. Revista Geográfica De América Central. 2 (47E): 1-15 pp.

Guzmán Chávez, M. y Juárez Bolaños, D. (2013). En busca del ecoturismo. Casos y experiencias del turismo sustentable en México, Costa Rica, Brasil y Australia. México: Ediciones EÓN Sociales.

Hasse, G. (1986). Theorethical and methodological foundations of landscape ecology, in: Landscape Ecology. Abstract of Lecture. International Training Course. Institute of Geography and Geoecology, GDR Academy of Science, Leipzig. 4-7 pp.

Herrera, W. (1985). "Clima de Costa Rica. Vegetación y clima de Costa Rica”. (Vol. 2). EUNED. San José, Costa Rica.

Holdridge, L. (1967). "Life Zone Ecology". Tropical Science Center. San José, Costa Rica. 149 pp.

Honey, M. (2008). Ecotourism and sustainable development: Who owns paradise?. $2^{\text {a }}$ ed. Washington: Ed. Island Press.

Huerta, M. y Sánchez, A. (2011). "Evaluación del Potencial Ecoturístico en Áreas Naturales Protegidas del Municipio de Santa María Huatulcu, México". Cuadernos de Turismo. 541-560 pp. 
Instituto Nacional de Estadística y Censos [INEC]. (2020). Empleo y Pobreza por línea de ingreso. Lugar de publicación: Página web del INEC. Disponible en: https://www.inec.cr/sites/default/files/documentos/inec_institucional/estadisticas/resultados/reeconomcenso2011-08.pdf.pdf

García, M. (2019). Comunicación personal y correo electrónico, 16 de julio 2019 (entrevista).

Morera, C. (2001). "Ecoturismo y Desarrollo local en la Península de Osa, Costa Rica”. Revista Geográfica de América Central. 1 (39): 41-74 pp.

Municipalidad de Sarapiquí. (2012). Información Poblacional. Obtenido de Municipalidad de Sarapiquí. Lugar de publicación: Página web de la Municipalidad de Sarapiquí. Disponible en: https://sarapiqui.go.cr/nuestra-municipalidad/ datos-generales/136-informacion-poblacional

Programa de las Naciones Unidas para el Desarrollo [PNUD]. (2011). Atlas del desarrollo humano cantonal de Costa Rica 2011. Lugar de publicación: Página web. Disponible en: https://www.undp.org/content/dam/costa_rica/docs/undp_cr_atlas_cantonal.pdf

Quesada, A. G. (2019). Conozca el osito más pequeño del planeta habitante de nuestros bosques. Lugar de publicación: El Norte Hoy. Disponible en: https://elnortehoy.wordpress.com/2019/12/31/conozca-elosito-mas-pequeno-del-planeta-habitante-de-nuestros-bosques/

Reserva Biológica Tirimbina. (s.f.). Tours. Lugar de publicación: Página web de Tirimbina. Disponible en: https:/www.tirimbina.org/tours/

Sarapiquí Sostenible. (2018). Generalidades del destino. Lugar de publicación: Sarapiquí Sostenible. Disponible en: https://sarapiquisostenible.com/generalidades-del-destino/.

Thornthwaite, C. (1948). "An approach toward a Rational Classification of Climate”. Geographical Re-view. 38(1): 55-94 pp.

Vega, A. (2014). Caracterización Territorio Sarapiquí. Lugar de publicación: INDER. Disponible en: https://www.inder.go.cr/sarapiqui/ Caracterizacion-Territorio-Sarapiqui.pdf

Weaver, D. y Lawton, L. (2007). "The state of contemporary ecotourism research". Tourism Management. 28 (2007): 1168-1179 pp. 
